\title{
MEMORIAS
}

\section{Reflexiones en torno a la formación de profesionales de la rehabilitación en el abordaje de la primera infancia}

DEPARTAMENTO DE INVESTIGACIÓN ECR

29,30 y 31 de agosto de 2018

\section{Presentación}

Durante los días 29, 30 y 31 de agosto del presente año se llevaron a cabo los eventos de del Departamento de Investigación de la Escuela Colombiana de Rehabilitación: "Jornadas de Ambientes Estudiantiles de Investigación", "Evento Semilleros de Investigación" y "VII Encuentro La ECR Investiga". Estos tuvieron como objetivo principal generar un espacio de intercambio de conocimiento y reflexión que permitiera comprender el abordaje e importancia de la primera infancia en las profesiones de rehabilitación con las entidades académicas y gubernamentales. La temática se priorizó debido a la importancia Nacional que está teniendo en las profesiones de salud y como parte de discusión del grupo Capacidades Humanas, Salud e Inclusión de la ECR.

Se contó con el apoyo de invitados internacionales de la Universidad de Andrés Bello de Chile y del Centro Universitario Mexicano de Humanidades y Ciencias de la Salud CMUCH.
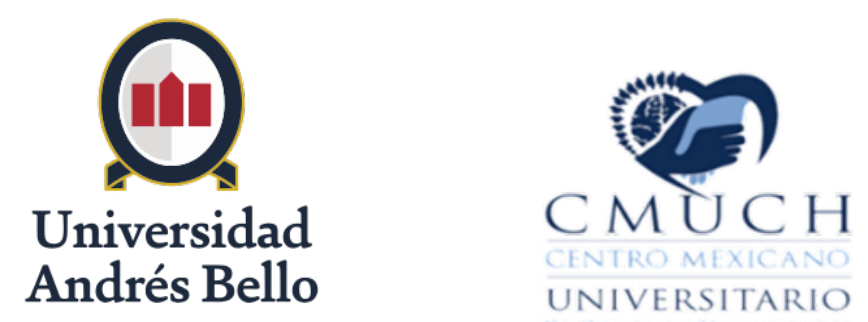

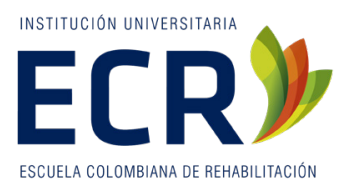

1. Organizó el Departamento de Investigación de la Escuela Colombiana de Rehabilitación bajo la coordinación de Olga Lucía Montoya Hurtado, directora del grupo de investigación Capacidades Humanas Salud e Inclusión y Carlos Alberto Pérez, Líder de Investigación Institucional de la ECR. Correo de contacto: omontoya@ecr.edu.co 


\section{Jornadas de Ambientes Estudiantiles de Investigación ${ }^{2}$}

La jornada de ambientes estudiantiles de investigación tuvo como objetivo visibilizar la articulación de las diferentes herramientas de investigación con el desarrollo de competencias en prácticas y pasantías. Se realizó la apertura por parte de una egresada que hizo evidente una experiencia en la incorporación de la investigación en la toma de decisiones en salud dentro de su práctica cotidiana. La egresada Johana Moreno Avendaño, ha logrado incorporar la investigación tanto para su práctica asistencial como para su formación en la maestría neurofisiología en la Universidad Nacional de Colombia. Presentó la ponencia titulada "La investigación como herramienta esencial para la toma de decisiones en la clínica". La experiencia presentada hizo evidente cómo la investigación ha aportado a la construcción personal y profesional de la egresada, y le ha permitido perfilarse en su práctica clínica y además, en un área de conocimiento de poco abordaje por los fisioterapeutas, como lo es la investigación básica en animales en neurofisiología.

Adicionalmente se presentaron tres ponencias con la participación de estudiantes de quinto y sexto semestre, con temáticas como "Consulta Comunitaria: Una experiencia de abordaje interdisciplinar", "La ocupación de juego como medio en la vinculación de la cultura tradicional Mhuysqa con la cultura occidental" y "Herramienta fonoaudiológica de entrenamiento de un sistema de comunicación alternativa con una aplicación móvil para usuarios en condición de discapacidad". Estas presentaciones lograron, a partir de experiencias de práctica, mostrar al auditorio la forma en que el uso de herramientas de investigación les permitió a los estudiantes una mejor toma de decisiones, el acceso a la información de personas y comunidades; y la programación de intervenciones acordes a las necesidades de individuos y colectivos. Las experiencias tienen la riqueza de la experiencia en comunidad (indígena) y además el desarrollo de la investigación en contextos clínico-terapéuticos, lo que además muestra la amplitud de campos de acción de las profesiones que se forman en la ECR.

Se finalizó la mañana con el conversatorio titulado "Intercambio de experiencias en investigación para el desarrollo de competencias disciplinares”. Para el conversatorio se contó con la participación de las estudiantes Ludy Vanegas, Mónica Tapiero, y Natalia Buitrago de los programas de Fisioterapia, Terapia Ocupacional y Fonoaudiología de la Escuela Colombiana de Rehabilitación; a la Rectora de la Escuela Colombiana de Rehabilitación, doctora Clara Patricia Giraldo Pulgarín; al coordinador de investigación de la facultad de ciencias de la rehabilitación de la Universidad Andrés Bello de Chile, doctor Daniel Jeréz Mayorga; al Rector del Centro Mexicano Universitario de Ciencias y Humanidades de México, doctor Marco Antonio Cubillo; y a la y la Vicerrectora del Centro Mexicano Universitario de Ciencias y Humanidades de México, doctora Rosa Martha Guillén.

El conversatorio tuvo un desarrollo de mucho interés para los organizadores, dado que colocaba en el mismo espacio de disertación a estudiantes de práctica de las tres facultades y además a la rectora de la ECR, al rector y vicerrectora del CMUCH, y al Coordinador de investigación de la facultad de ciencias de la rehabilitación de la Universidad Andrés Bello de Chile. Esto implicó un intercambio interesante de experiencias e intencionalidades puesto que los estudiantes lograron de forma estructurada mostrar los beneficios, las dificultades y los retos que ha sido aplicar la investigación en la toma de decisiones basada en la evidencia en los diferentes contextos de práctica, pero además, desde los profesores participantes, se lograron hacer evidentes los propósitos que tienen las instituciones de educación superior cuando incorporan dentro de los planes de estudio núcleos de investigación muy completos. Este espacio particular logró discursos de alto nivel en el que se reconocieron los momentos exitosos en el uso de la investigación en el logro de competencias, pero también como los momentos de dificultades generan espacios de creatividad que llevan a los estudiantes a explorar nuevas opciones para la resolución de conflictos a partir de la investigación.

En la tarde se realizó el Taller liderado por el Centro Nacional de Memoria Histórica titulado "Abordaje en niños víctimas del conflicto". Este espacio permitió desde la narración de dos investigadoras del Centro de Memoria Histórica, reconocer las formas de comprender las realidades asociadas al histórico conflicto armado en Colombia y de vincular a 
las comunidades a la solución de sus diversas problemáticas. Las investigadoras mostraron los diversos acercamientos metodológicos a la infancia desde la investigación cualitativa, y cómo a partir de la comprensión de los diferentes determinantes del conflicto, lograron generar transformaciones en las comunidades, por medio de estrategias centradas en las raíces culturales de las diferentes víctimas del conflicto.

Al cierre del evento, fue evidente el cumplimiento de los objetivos, y de cómo poco a poco se ha logrado que los estudiantes de la Escuela Colombiana de Rehabilitación logren hacer conciencia de la investigación como medio para acercarse a las realidades, de cómo una pregunta adecuada permite generar las condiciones precisas para responder adecuadamente a las necesidades de personas o colectivos
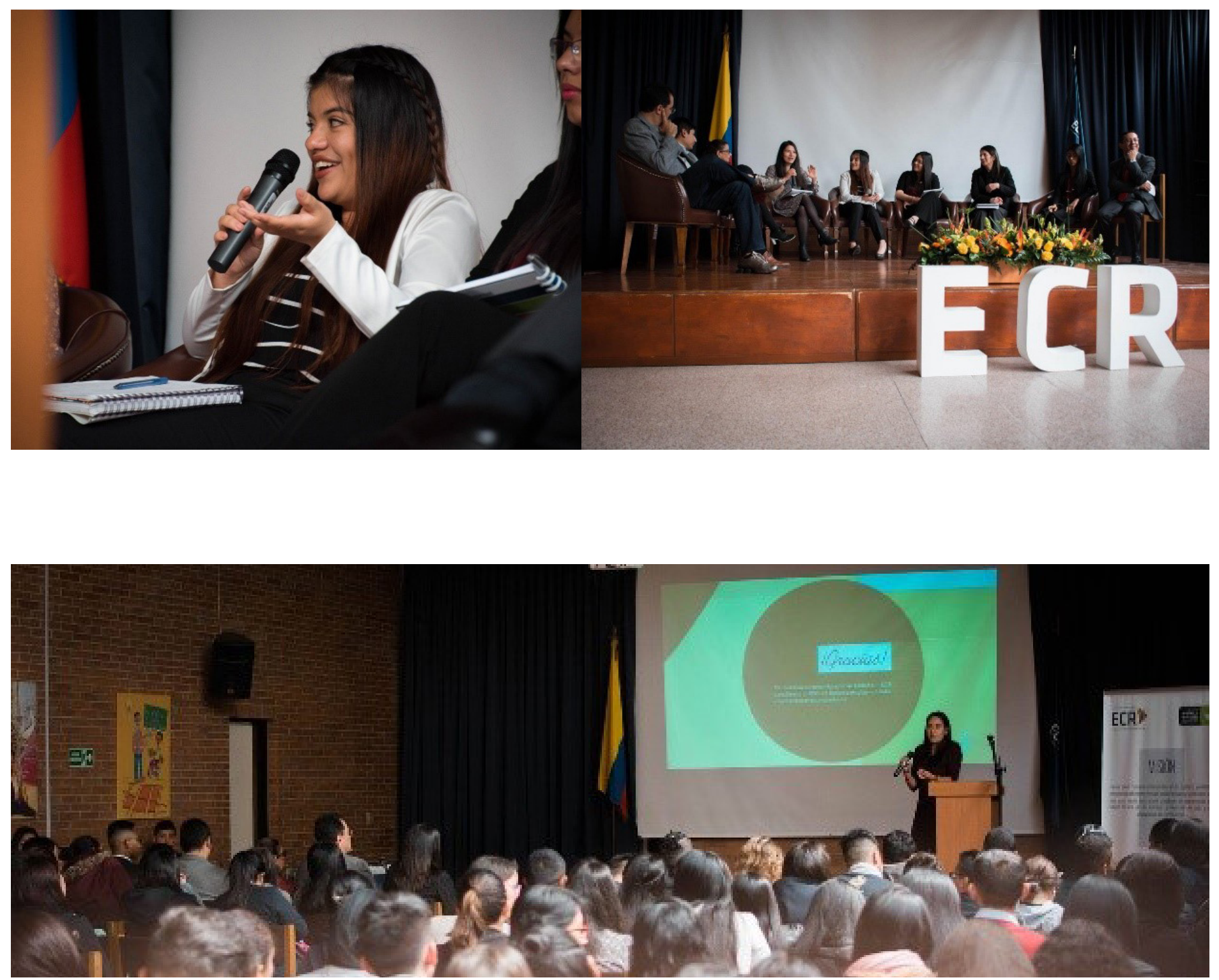

2. Texto de memoria realizado por el profesor Carlos Alberto Pérez - Líder de Investigación Institucional-ECR 


\section{Evento Semilleros de Investigación ${ }^{3}$}

Para el evento de semilleros de Investigación se contó en la jornada de la mañana con la presentación de 6 ponencias y 10 ponentes. Todas las temáticas giraron en torno a cómo los semilleros de las profesiones de rehabilitación están investigando en temáticas de infancia. Se presentaron temáticas como "Plan de entrenamiento para la población escolar que utiliza tecnología de apoyo-prótesis-de miembro superior, "Identificación y Seguimiento de Signos de Alarma del Desarrollo Psicomotor mediante un programa de telecuidado", "El rol del fisioterapueta en la rehabilitación en las alteraciones del Neurodesarrollo asociadas a la infección con virus ZIKA", " Lugar del fisioterapeuta en los lineamientos para la primera infancia en Colombia", "Cómo enfrentar el ciber acoso sexual infantil en niños, niñas y adolescentes: lineamientos para su prevención", "Recorridos investigativos: vinculaciones desde la experiencia académica como aprendices y como profesionales en torno a la literatura sorda” y representantes de facultad de psicología de la Universidad Católica de Colombia perteneciente al Semillero de Investigación identidad y trabajo del área organizacional, semillero de investigación Ocuparte de la ECR, de la especialización de fisioterapia en neurorehabilitación integrante del semillero Kinésis, semillero en Neurociencias del departamento de ciencias básicas de la ECR , programa de fisioterapia de la UNAL y presidencia de la Asociación Colombiana de Estudiantes de Fisioterapia, programa de psicología de la Universidad Católica de Colombia del Semillero de investigación Identidad y trabajo del área organizacional y semillero de investigación Cognición y Lenguaje de la UNAL.

Los invitados internacionales escucharon durante la mañana las ponencias y después participaron en un conversatorio liderado por uno de los coordinadores de semillero de la ECR donde quedó como reflexión para el abordaje en primera infancia tener en cuenta la complejidad y trabajo trasndisciplinario. Los invitados internacionales recomendaron seguir fomentando la cultura y amor por la investigación.

En la tarde se presentaron los talleres a cargo del Dr. Daniel Jerez de Chile Universidad Andrés Bello: "Uso de las Tecnologías de bajo costo en Rehabilitación” y “Descripción del modelo de Intervención en el Instituto Transdisciplinario de Rehabilitación” a cargo del Dr. Marco Antonio Cubillo de México Universidad de CMUCH, los participantes manifestaron gran interés por la tecnología y aplicación del modelo transdisciplinario de rehabilitación.

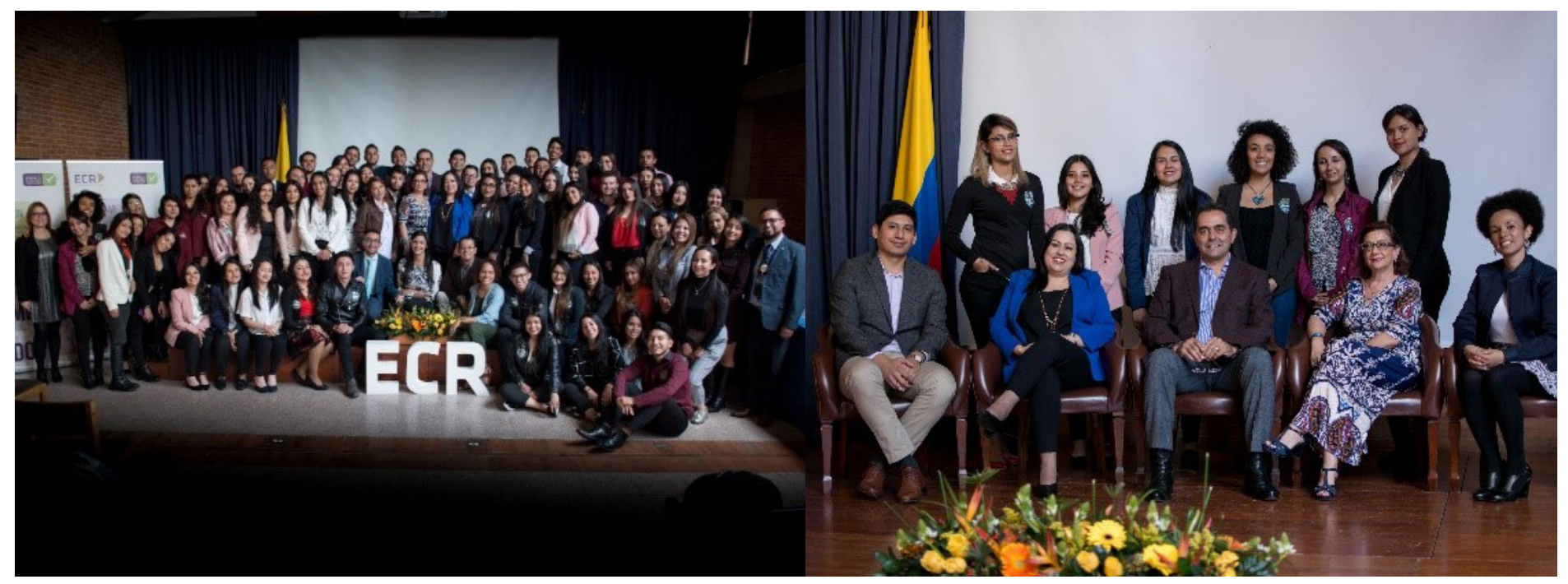

3. Texto de memoria realizado por la profesora Olga Lucia Montoya Hurtado- Directora Grupo de Investigación Capacidades Humanas, Salud e Inclusión ECR 


\section{Encuentro la Ecr Investiga ${ }^{4}$}

Para el desarrollo del evento de la ECR investiga, teniendo en cuenta que era el último día de las jornadas de investigación, se realizó la apertura con las palabras de la Dra. Clara Patricia Giraldo, Rectora ECR y Gloria Isabel Bermúdez, Jefe Departamento de Investigación ECR. En la jornada de la mañana se presentaron cuatro ponencias, tres internacionales y una nacional, la ponencia de Colombia "Importancia de la política de estado de cero a siempre en el abordaje del desarrollo integral de la primera infancia: el lugar y la voz de las profesiones de Rehabilitación" estuvo a cargo del Dr. Cesar Luna Vivas, representante de la Subdirección de gestión técnica para la atención a la primera infancia- Dirección de Primera Infancia.

Posteriormente, se presentaron las ponencias internacionales de los países invitados, México: "Hacia un Modelo de Habilitación y/o Rehabilitación Transdisciplinaria”, a cargo del Dr. Marco Antonio Cubillos, Centro Mexicano Universitario de Ciencias y Humanidades; Chile: "Tecnología en Rehabilitación” a cargo del Phd. Daniel Alejandro Jerez Mayorga, Universidad Andrés Bello; y México: “Intervención Tanatológica sobre los Procesos de Pérdida”, a cargo de la Mtra. Rosa Martha Guillén Vice Rectora Universidad de CMUCH.

Al finalizar la mañana se realizó el conversatorio ICBF - Asociaciones Gremiales- Invitados Internacionales, liderado por la docente experta en primera infancia PhD. Carolina Soler de la Universidad pedagógica de Colombia. En este conversatorio se dio una reflexión importante en cuanto a unir esfuerzos gremiales para participar en la construcción de política pública en infancias, participación de las profesiones de rehabilitación en las rutas de atención primaria y en la construcción de los cursos específicos para el abordaje integral en infancias.

\section{Conversatorio}

La infancia y la formación de profesionales en el campo de la rehabilitación.

Moderadora: Dra. Carolina Soler Martín ${ }^{5}$

Universidad Pedagógica Nacional de Colombia.

El día 31 de agosto de 2018, en el marco del VII Encuentro Internacional la ECR Investiga. Experiencias en la formación de profesionales de rehabilitación en primera infancia, organizado por el Departamento de Investigación y el Grupo de investigación Capacidades Humanas, Salud e Inclusión, se desarrolló el conversatorio titulado: Reflexiones en torno a la infancia y la formación de profesionales en el campo de la rehabilitación. En este, participaron invitados pertenecientes a las agremiaciones nacionales de las profesiones de Terapia ocupacional, Fisioterapia y Fonoaudiología junto con los invitados del Instituto Colombiano de Bienestar Familiar y de dos Universidades internacionales que desarrollan investigación en el campo de la rehabilitación ${ }^{6}$.

El conversatorio tuvo como objetivos explorar con los invitados desde sus trayectorias de vida y profesionales, dos aspectos:

a) Aquellas experiencias que impulsaron el interés por el trabajo desde la rehabilitación con los niños, niñas y la infancia $y$,

b) las tensiones o preocupaciones vigentes que, con estos mismos sujetos, conciernen a la formación de profesiona les en el campo de la rehabilitación.

4. Texto de memoria realizado por la profesora Olga Lucia Montoya Hurtado- Directora Grupo de Investigación Capacidades Humanas, Salud e Inclusión ECR

5. $\mathrm{PhD}$ en Ciencias Sociales. Coordinadora Maestria en Estudios en Infancia. Univerisidad Pedagógica Nacional 
Lo anterior, con el fin último de exaltar pistas o indicios, construidos o a construir, tanto en los ámbitos curriculares como investigativos en las instituciones formadoras de profesionales de la rehabilitación, que posibiliten avanzar, por una parte, en las articulaciones con las re alidades de los niños y las niñas, así como con las instituciones encargadas de su atención y con las políticas públicas; y por otra, en la construcción del campo de conocimiento de cada una de las profesiones en rehabilitación y, en las relaciones con otras profesiones.

Definir los anteriores aspectos fue resultado del diálogo entre los organizadores del conversatorio, quienes coinciden en que la infancia o, las infancias, si se quiere dar cuenta de la multiplicidad de referentes y realidades construidos alrededor de los niños y niñas, ha tomado fuerza en la agenda política y pública de nuestra sociedad hace algunas pocas décadas. Igualmente, que es un campo de investigación y desarrollo que exige construcciones teóricas y prácticas en las diferentes profesiones y, por ende, en la formación de profesionales convocados al encuentro y trabajo con los niños y niñas. Algunas palabras que recogen dicho diálogo para la consolidación de las preguntas, pueden ser las expuestas por Cosse, Llovet Villalta y Zapiola (2011):

(...) la "infancia" no puede ser vista como un descriptor aproblemático de una fase natural. Por el contrario, debe verse como una expresión cultural particular, histórica, políticamente contingente y sujeta a cambios (...) la manera en que vemos y actuamos respecto de los niños no es inocua. Por el contrario, esa mirada da forma a la experiencia infantil, así como a sus propias respuestas y a su involucramiento con el mundo adulto. (p. 12)

\section{Reflexiones derivadas del conversatorio}

Tras la presentación realizada por cada uno de los invitados al conversatorio, se pueden comprender tres escenarios en las cuales se han dinamizado intereses o hechos que han llevado a las profesiones de la rehabilitación a trabajar con los niños y las niñas así como a dialogar y aportar a la categoría de infancia. Escenarios que también entrevén tensiones, diferentes fuerzas y problemáticas que confluyen en estas profesiones, comprendiendo la multiplicidad de perspectivas, realidades y exigencias a las cuales se ven convocadas.

\section{El mandato social del trabajo con los niños y niñas en las profesiones de la rehabilitación}

Un primer escenario en el cual acuerdan algunos de los invitados es innegable la presencia y trabajo con los niños y niñas, se fundamenta en una perspectiva socio-histórica y se corresponde con las demandas y apuestas que en los comienzos de la formación de profesionales en Fisioterapia, Terapia Ocupacional y Fonoaudiología, la sociedad del momento realizó a estas. Para el caso colombiano entre los años cincuenta y setenta, las necesidades sociales, proyecciones institucionales y algunas políticas, implicaron inmediatamente a estas profesiones en el trabajo rehabilitatorio de niños y niñas. Puede ser útil la noción de "contrato fundacional"7 para comprender este asunto, es decir, qué esperaba la sociedad que cumpliera cada profesión e institución creada en determinado momento histórico, cultural y político.

El contexto colombiano de las décadas mencionadas, guiado por tendencias internacionales y avances en el campo de la medicina y la rehabilitación, inicia la institucionalización de la formación de profesionales en esta última área. Una de las demandas sociales centrales realizadas a las instituciones formadoras, fue el trabajo con niños y niñas con diferentes diagnósticos clínicos, patologías, necesidades de atención, desescolarizados, principalmente desde prácticas individuales y bien fuera situados en instituciones clínicas, educativas o de protección.

6. .Dra. Rosa Martha Guillén y Dr. Marco Cubillo del Centro Mexicano Universitario de Ciencias y Humanidades de México; Dr. Daniel Jerez de la Universidad Andrés Bello de Chile; MSc. Cesar Luna del Instituto Colombiano de Bienestar Familiar; Dra. Ayde Luisa Roballo Torres del Colegio Colombiano de fisioterapia; Esp. Diana Marcela Laverde Robayo del Colegio colombiano de fonoaudiología; y MSc. Clemencia Martínez Londoño, del Colegio Colombiano de Terapia Ocupacional. 
En sus inicios, las carreras de rehabilitación, de preferencia femeninas como indica una de las invitadas al conversatorio y con carácter técnico, centraron sus perspectivas en la enfermedad, la terapia y la noción tradicional de rehabilitar, esto, en tanto la cercanía, o dependencia, con los referentes médicos. Además, en el contexto inicial de estas profesiones se demandó su labor de servicio social y asistencial con los niños y niñas, asunto central a mediados del siglo pasado ante las consecuencias de la violencia en el país y dado que aún no se instalaba la perspectiva de derechos.

Exaltar que en el país, desde los inicios de la formación de profesionales en rehabilitación se ha exigido el trabajo con los niños y las niñas, invita a proponer algunas preguntas que si bien no fueron abordadas en el conversatorio, pueden ser pertinentes para nuevos encuentros y acercan a la comprensión de tensiones en la formación, por ejemplo, en el país ¿Desde qué referentes epistemológicos, construidos y transitados, las profesiones en rehabilitación y las instituciones formadoras se han aproximado a las infancias? ¿Cuáles han sido y cómo se han construido los diálogos entre profesiones de la rehabilitación y, entre estas con otras profesiones, cuando se centran en los procesos de atención de niños y niñas?

\section{Tendencias internacionales, nacionales y apuestas de los programas de formación}

Un segundo escenario, cohesionado con el anterior, se relaciona con las oportunidades que los invitados vivieron en su formación profesional en las distintas instituciones formadoras. Bien sea oportunidades curricularizadas tales como prácticas, cátedras, investigaciones, o bien encuentros con otras profesiones, lecturas, eventos así como otras experiencias, las miradas hacia los niños y niñas hicieron parte de los años de formación como profesionales.

Los invitados señalan experiencias de formación puntuales, por ejemplo, prácticas durante varios semestres en las cuales debían desarrollar proyectos de atención, de desarrollo social o de investigación desde cada uno de sus campos de conocimiento. Esta oportunidad les exigió comprender algunos referentes teóricos que circulaban en su momento así como las estrategias y prácticas rehabilitatorias derivadas de estos. Pasados los años, esos acercamientos y la reflexión sobre los mismos, posibilitan analizar los cambios en los sentidos, terminología, intenciones y el ejercicio de la profesión junto con o, para los niños y niñas.

También, señalan influencias de las políticas y normativas nacionales e internacionales y sus direccionamientos e influencias en los currículos de formación.

Indican, por ejemplo, cuando fueron estudiantes en las décadas de los ochenta y noventa, cómo algún profesor abordó las políticas sobre derechos en relación con los niños y niñas, alguno problematizó realidades y relaciones culturales o coyunturas sociales en comunidades específicas o, en años más recientes, también ellos pero ahora como profesores, introdujeron en las clases referentes como enfoque poblacional, política pública, tecnología y rehabilitación, inclusión y otros que se instalan de diferentes formas en los sujetos, las instituciones y los discursos y que, por ende, configuran las historias vividas.

El interés en el país por la historia de las profesiones en rehabilitación ha conllevado a diferentes estudios y análisis que circulan en el medio académico, cada uno desde lugares de enunciación y alcances particulares. Derivado de este interés y como contribución de algunos sucesos y recreaciones de los invitados al conversatorio, se arriesgan algunas preguntas para continuar el debate: ¿Cuáles son las tensiones y tendencias teóricas y prácticas en relación con las infancias, que en el ámbito curricular están presentes en la formación de profesionales de la rehabilitación? ¿Cuáles son las condiciones de posibilidad en la formación de estos profesionales, para la reflexión y construcción de conocimientos situados desde las relaciones rehabilitación-infancias?

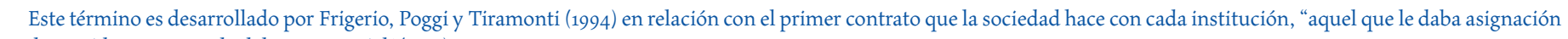
de sentido a esa parcela del campo social" (p. 11). 


\section{Experiencias y acontecimientos con la infancia, en la infancia y los caminos recorridos.}

Un tercer escenario, desde una perspectiva instituyente y por consiguiente con afectación en los trayectos de vida, se refiere a experiencias personales, en ocasiones individuales e íntimas que cursaron y siguen cursando un eje de reflexión que involucra la propia infancia y la de los otros. Esta connotación de experiencia personal no excluye a las instituciones; allí, como en cualquier escenario, se construyen subjetividades junto con los otros.

Algunos de los invitados afirman recordar, reflexionar o comparar la infancia vivida o de aquellas de las que se tiene referencias, y las formas como estas incidieron y contribuyeron a ser lo que se es y a hacer lo que se hace hoy en día. Parafraseando a Jorge Larrosa, el asunto de la experiencia se refiere a "eso que me pasa" y luego de lo cual no soy el mismo. Y les pasaron a los invitados, por ejemplo, encuentros generacionales, viajes, amistades, problemas, autores, películas, con los cuales cobra sentido la frase que algunos de ellos más o menos enunciaron: "la vida me fue llevando a trabajar con los niños y niñas".

Si bien el conversatorio mantuvo la intención de dialogar sobre relaciones entre las profesiones en rehabilitación y la infancia, el asunto de las experiencias personales son génesis, trascienden y en ocasiones dan giros a los roles asumidos. El poder narrarse en relación con la propia infancia y con los niños y niñas que interpelan la vida misma, y no solo narrarse desde la rehabilitación, exalta relaciones entre maestros y estudiantes, padres e hijos, abuelos y nietos, por ejemplo, que se construyen desde otros lugares, relaciones que son destacadas por algunos de los invitados como un asunto central en la vida que, en ocasiones o, siempre, influyen en el ejercicio de la profesión.

Los comentarios de los invitados que dieron posibilidad para comprender las relaciones entre sus caminos de vida y el trabajo con, por y para los niños y niñas, comentarios apenas dados como pinceladas porque el tiempo en un conversatorio no juega a favor $\mathrm{y}$, siempre hay tanto para decir, invitan a retomar preguntas profundas y de larga data para las instituciones educativas, una de estas podría ser ¿A cuál sociedad estamos dirigiendo nuestras propuestas formativas? ¿Cuál sociedad soñamos? Y desde allí, ¿Qué lugares queremos construir en relación con los niños y niñas y con las nociones de infancia?

Las instituciones formadoras de profesionales, como posibilitadoras de experiencias que no nos dejan igual a como éramos, están llamadas a acoger, a dar lugar a experiencias de vida y a construir otras más, pero además, a construir las herramientas para reflexionar sobre estas, pues la reflexión es una oportunidad para construir sueños individuales y colectivos de sociedad.

Lo anterior, abre nuevos cuestionamientos para seguir pensando y actuando desde las instituciones formadoras, por ejemplo ¿Cómo aprovechar en la formación de profesionales de la rehabilitación las oportunidades de vida con los niños y niñas, la disposición de los estudiantes, de muchos, para el trabajo con ellos?

Por último, y a manera de cierre, las instituciones laborales en las cuales se encuentran los invitados, siendo estas Universidades e Instituciones de protección, y por otra parte las agremiaciones y redes de pertenencia, han respondido a las necesidades de investigación, gestión y desarrollo emanadas de las políticas, se ven influenciadas por las tendencias y presiones internacionales y nacionales en los ámbitos culturales, sociales, políticos y económicos, comprenden que la vinculación con los niños, niñas y las infancias refieren aspectos históricos tanto sistemáticos como coyunturales y, reconocen que tienen una amplia responsabilidad en la construcción continua del campo de conocimientos de cada una de las profesiones en rehabilitación. 
En la jornada de la tarde se realizaron tres talleres en paralelo, Taller "Descripción del modelo de Intervención en el Instituto Transdisciplinario de Rehabilitación" México, Universidad de CMUCH, Taller "Uso de Aplicaciones Móviles para la Rehabilitación" Chile Universidad Andrés Bello Taller "Sexualidad en las Personas con Discapacidad" México, Universidad de CMUCH donde participo uno de los docentes y líder de la línea de diversidad e inclusión del grupo de investigación de la ECR.

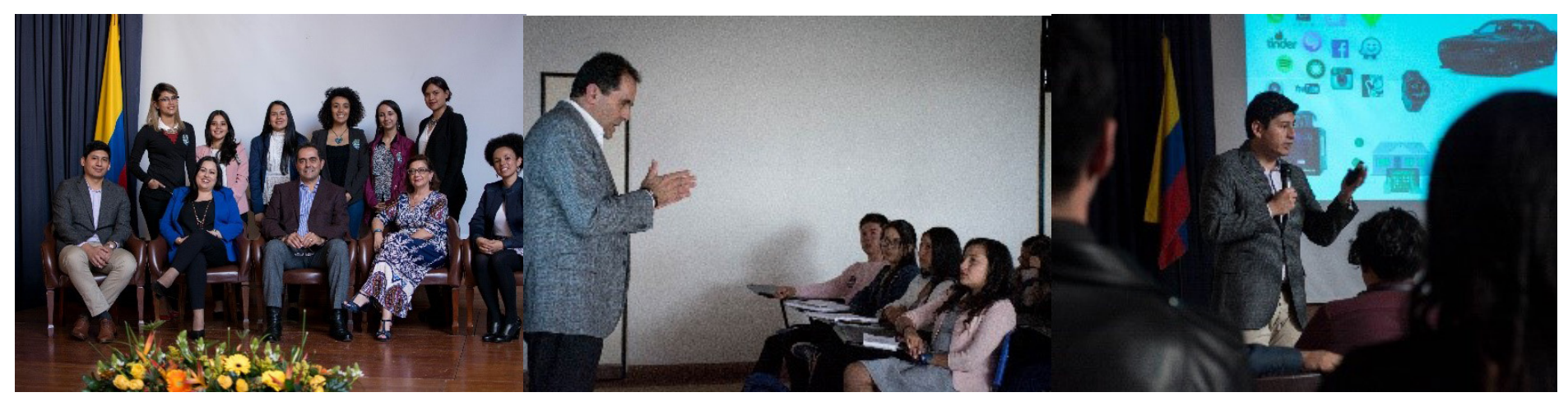

\section{Ponencias}

Hacia un Modelo de Habilitación y/o Rehabilitación Transdisciplinaria "Por un cambio de paradigma: de la disciplina a la Transdisciplina"

Dr. Marco Antonio Cubillo León ${ }^{8}$

Centro Mexicano Universitario de Ciencias y Humanidades

Hoy entendemos que la discapacidad es un fenómeno complejo que nos obliga a reconocer que tenemos que apoyarnos en diferentes ciencias y disciplinas para poder analizarla, interpretarla y consecuentemente establecer las mejores estrategias de intervención de habilitación y rehabilitación orientadas a la persona con discapacidad o en riesgo de padecerla.

Dentro de los problemas que presentan las personas con discapacidad destacan las repercusiones que involucran no solo a su contexto biológico-psicológico-espiritual personal sino también a su contexto familiar y social. Y todos ellos se tienen que interpretar de acuerdo a toda una serie de determinantes culturales, sociales, económicas, laborales, de salud, espirituales, familiares y mentales con toda su amplia gama de posibilidades e interacciones.

Hemos de reconocer que la discapacidad es un fenómeno complejo cada vez más frecuente en la población mundial y, a lo largo del ciclo de vida, ha de presentarse con una serie de particularidades que tenemos que ser capaces de entender de acuerdo a la etapa de la vida en que la persona la padece.

Por otra parte, la persona con discapacidad requiere ser atendida por profesionales que tengan la formación necesaria para atenderle de manera integral.

Por estas razones, se hace indispensable tener la posibilidad de generar y crear las mejores estrategias de intervención que nos permitan atender y acompañar al paciente durante todo su proceso de habilitación o rehabilitación, es decir desde que éste empieza hasta lograr su inclusión plena dentro de su contexto personal, familiar, escolar, laboral, social.

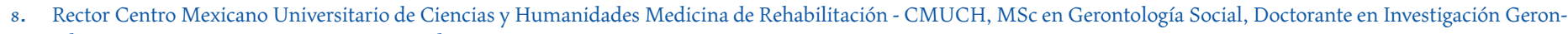
tológica, Doctorante en Pensamiento Complejo. 
Hemos establecido un modelo de atención con un enfoque transdisciplinario, lo que nos permite integrar las opiniones de todos los miembros del equipo de intervención que incluye médicos generales y especialistas en rehabilitación, medicina del deporte, neurología, psiquiatría, ortopedia; así como expertos en psicología, neuropsicología, fisioterapia, terapia ocupacional, terapia del lenguaje, gerontología, arteterapia e ingeniería biomédica con el fin de generar un programa integral que permita conducir a la persona con discapacidad y a su familia a lo largo del camino que inicia desde el momento en que se hace necesario empezar trabajar con él, sea dentro o fuera de un hospital, y hasta lograr su plena inclusión dentro de su entorno social.

\section{La Educación Tanatológica como parte integral en la formación de Profesionales”}

Rosa Martha Guillén Apreza ${ }^{9}$

Centro Mexicano Universitario de Ciencias y Humanidades

¿Qué es la Muerte?, ¿Cómo la visualizo?, ¿Qué siento al hablar de mi propia muerte?. Estas son las preguntas que más temor nos causan, por lo que representa mi propia muerte. ¿Acaso no me he dado cuenta de que el hombre es un ser finito, que desde que nace ya trae a la muerte consigo?

¿A qué le tememos? ¿A la muerte en sí, o al propio acto de morir? ... La Educación Tanatológica, nos ayuda a encontrar las respuestas a estas preguntas, de aquí la importancia de conocer el concepto de Tanatología.

Tánatos=muerte $\operatorname{logo}=$ tratado, estudio, discurso

Ciencia encargada de encontrar sentido al proceso de la muerte y las pérdidas que conlleva.

Desde el momento en que nacemos, empezamos a morir, pero ese pensamiento está muy lejos de nuestro presente, desde pequeños, en muchas culturas no se nos permite vivir de cerca la muerte física, se nos esconde el acto de morir y, dependiendo de esta experiencia es como cada uno de nosotros conforma esa representación social o constructo social acerca de la muerte.

Por lo anterior, todos debemos accesar a una Educación Tanatológica, que nos ayudará a comprender y procesar el significado de la muerte y las pérdidas que nos rodean. El miedo siempre estará con nosotros, pero el cómo lo manejemos tiene mucho que ver en cómo entiendo su significado.

La muerte es universal, individual y no conoce edad. De acuerdo al contexto socio-cultural-histórico en que nos toca vivir, realizamos de manera inconsciente el constructo social de ese concepto.

La educación tanatológica es indispensable en la formación integral del ser humano; ésta ayudará a encontrarle un sentido a la vida. Si reconozco y acepto mi propia muerte, entonces disfrutaré el día a día valorando lo que soy y lo que tengo

\section{Tecnología en Rehabilitación}

Dr. Daniel Alejandro Jerez Mayorga ${ }^{10}$

Universidad Andrés Bello de Chile

El acceso a la tecnología es cada vez mayor, por lo que hoy en día el uso del celular para comunicarnos es una práctica rutinaria y habitual. En el ámbito de la rehabilitación, salud y del rendimiento deportivo el uso de aplicaciones móviles ha tenido un auge en los últimos años siendo esta utilizada como un método de control y monitoreo de diversas variables mecánicas, fisiológicas, calidad de vida y rendimiento físico.

9. Vicerrectora Centro Mexicano Universitario de Ciencias y Humanidades Medicina de Rehabilitación - CMUCH. MSc. en Gerontología Social 
Algunas de estas aplicaciones móviles presentan una gran confiabilidad y validez cuando han sido comparadas con el "Gold estándar" y por lo tanto son una herramienta costo efectiva para registrar y controlar datos de nuestros usuarios y/o deportistas. A través de estas podemos evaluar variables como la frecuencia cardiaca por medio de fotopletismografía, fuerza muscular, saltabilidad, mecánica de carrera a través del análisis de video, rango de movimiento y velocidad utilizando el giroscopio y el acelerómetro incorporados en estos.

Los niveles de actividad física, conducta sedentaria y gasto energético son variables que han sido ampliamente estudiadas utilizando aplicaciones móviles creadas especialmente para la recolección y análisis de estas variables. Por otra parte, el uso de otro tipo de tecnología de bajo costo como por ejemplo la plataforma Wii Balance Board ha demostrado ser una herramienta útil en la evaluación de la fuerza muscular isométrica en sujetos adultos mayores, además de entregar la opción de evaluar y registrar el balance, desplazamiento del centro de presiones y las fuerzas ejercidas en gestos funcionales como es el parar y sentarse de una silla.

Es importante tanto para el clínico como para el profesional de la rehabilitación contar con herramientas que nos permitan objetivar y medir de forma válida y fiable los diversos componentes de la función física.

\section{Importancia de la política de estado de cero a siempre en el abordaje del desarrollo integral de la primera infancia: el lugar y la voz de las profesiones de rehabilitación"}

Cesar Luna Vivas ${ }^{11}$

Instituto Colombiano de Bienestar familiar

Esta ponencia tiene como propósito dialogar en relación a los roles, responsabilidades y lugares que la ha dado la política de estado "De cero a siempre" a los y las ciudadanas en relación a la atención y protección de las infancias.

Nos habla de la corresponsabilidad, pero también de los entornos donde acontece la vida de los niños y las niñas, donde ocurren interacciones, se potencia el desarrollo y se favorece la inclusión de narrativas que tomen distancia de la mirada sobre los niños y las niñas asociada a la precariedad, la necesidad y la carencia.

Hablamos de un momento crucial en la historia, donde hemos logrado que converjan corrientes desarrollistas sobre las infancias, pero donde el espacio y el tiempo llaman a la convergencia de esfuerzos, en búsqueda de equidad en el acceso a los derechos de primera, segunda y tercera generación para los niños y las niñas.

Se plantean retos con una visión más allá de lo institucional y por ende, un abordaje multidisciplinar que ponga en sinergia la intervención en el ámbito de la rehabilitación con el ámbito de la protección, el cuidado y el desarrollo que recomienda la política de estado "De cero a siempre".

10. Director de investigaciones de la Facultad de Ciencias de la Rehabilitación. Universidad Andrés Bello de Chile

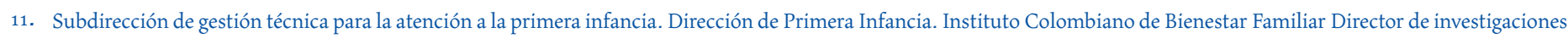
de la Facultad de Ciencias de la Rehabilitación. Universidad Andrés Bello de Chile 


\title{
Reflexiones y retos centrales
}

\author{
Diana Rocío Vargas Pineda \\ Nydia Alejandra Jiménez ${ }^{12}$ \\ Escuela Colombiana de Rehabilitación
}

El evento desarrollado, de carácter internacional, permitió que se suscitaran una serie de reflexiones y retos derivados de las presentaciones realizadas por los ponentes, del conversatorio en el cual practicaron especialmente representantes gremiales y de los talleres realizados, lo cual está en sintonía con las discusiones propias de la Línea Diversidad e Inclusión del Grupo Capacidades Humanas, Salud e Inclusión.

Existen varios retos qué contemplar a la hora de reflexionar alrededor de las temáticas que confluyen en el evento: la formación de profesionales de la rehabilitación y la primera infancia. En primer lugar, el pensar en la infancia implica hacer un ejercicio reflexivo sobre la comprensión actual de la niñez, que ha posibilitado entender a los niños y las niñas como sujetos políticos que se transforman y transforman la realidad, reconociendo todas sus capacidades y potenciales.

Vale la pena como seres humanos y como profesionales de la rehabilitación, considerar lugares, encuentros y personas que están presentes en el día a día de los niños y las niñas: los profesionales, la familia, sus pares; esto nos permite hablar de infancias, en plural.

Respecto a la política de Cero a Siempre, como profesionales y estudiantes, debemos apropiarnos de la misma y tener claridad respecto a su ejecución en el territorio, en la comunidad y en la cotidianidad, en espacios de salud, educación, bienestar, así como en el de participación política. Por tratarse de una política de Estado, es vital identificar la ruta de asignación de presupuestos, ya que desde nuestro quehacer profesional, muy posiblemente seamos quienes tengan la responsabilidad de agenciar planes, programas y proyectos alrededor de la misma.

El conocimiento y dominio que tengamos de esta vehiculiza tanto acciones derivadas de la política, como la asociatividad de la comunidad, lo que lleva a que posibilitemos la transformación de las prácticas sociales y culturales que no posibilitan el logro de los derechos de la infancia y nos lleva a pensar qué tanto se promueven los derechos de los niños y las niñas, así como el acompañamiento a eso procesos de vida, pero también, nos permite pensar también, que más que profesionales, somos seres políticos.

En cuanto al proceso de formación de profesionales en el campo de la rehabilitación, es de anotar que este ha estado permeado por experiencias de externos, por posturas conceptuales, metodológicas y en general comprensiones eurocentristas que distan de nuestra realidad Latinoamericana; por eso, hablamos hoy de la decolonización y de la necesidad de dar respuestas propias desde nuestras realidades, como es el caso de las epistemologías del sur: nuevas formas de conocer y teorizar. Se trata de confiar en nuestros propios desarrollos y ponerlos en marcha; se trata también de ser más que profesionales con un cúmulo de conocimientos que busca normalizar a los niños y las niñas, profesionales que desean ampliar las comprensiones de infancia, normalidad, salud e inclusión social.

Un ejercicio a recuperar tanto en la formación académica en el contexto de la práctica, como en la misma práctica profesional, es el acudir a herramientas que reconozcan las miradas comunitarias como la narración, las historias propias de niños y niñas, así como la identificación conjunta de necesidades. Las herramientas narrativas y aquellas que se brindan en la formación investigativa en el caso particular de la ECR, por medio de los cursos de investigación, son fundamentales y necesarias para acercarse a las realidades de los territorios, para comprenderlas y posibilitar las transformaciones necesarias. 
Esta percepción es compartida por los estudiantes de la ECR, quienes al acercarse al contexto de la práctica, valoran aquellas competencias y saberes adquiridos en estos cursos, los cuales son puestos en escena y les permite prepararse para la experiencia de trabajo comunitario.

Otro elemento a reflexionar consiste en pensar el desarrollo infantil de manera lineal, como resultado de una secuencia y así mismo plantear las intervenciones en rehabilitación desde nuestro quehacer profesional. Solemos basarnos en el enfoque biopsicosocial para sustentar nuestra aproximación a la población y en miradas aún mecanicistas de la salud y la rehabilitación. Si bien el enfoque biopsicosocial impulsado por medio Clasificación Internacional del Funcionamiento y la Salud aporta a la comprensión tanto de las estructuras y funciones de la persona, como de la participación, limita la puesta en escena de otros marcos que permiten hablar de la garantía de los derechos de niños y niñas y del empoderamiento y agenciamiento que puede llevar a cabo la misma familia, los cuidadores y/o el mismo contexto comunitario para abordar los problemas y necesidades más frecuentes en la infancia.

El enfoque de derechos es un marco interesante que complementa el enfoque biopsicosocial ya que implica dar poder al otro, es decir: empoderar, posibilitar el agenciamiento, y la corresponsabilidad, de los niños, las niñas y sus familias. Es de recordar que los usuarios, los pacientes y las comunidades tienen un saber previo y particular y desde ahí podemos valorar y potenciar ese conocimiento contemplando los principios del enfoque de derechos. Con esta mirada más integral, estamos traspasando fronteras del conocimiento y posibilitando diálogos con otras disciplinas, lo cual se puede ver reflejado en una propuesta de semillero transdisciplinar de investigación en rehabilitación y en la propuesta de una Red de infancia pensada desde la rehabilitación.

Finalmente, las reflexiones y retos presentados plantean una re significación del que hacer del profesional de la rehabilitación, transitando de lo reflexionado a acciones reales, derivando en un mayor posicionamiento profesional que inicia en la formación, y en una real participación e inclusión de los usuarios, los pacientes y las comunidades a los cuales abordamos.
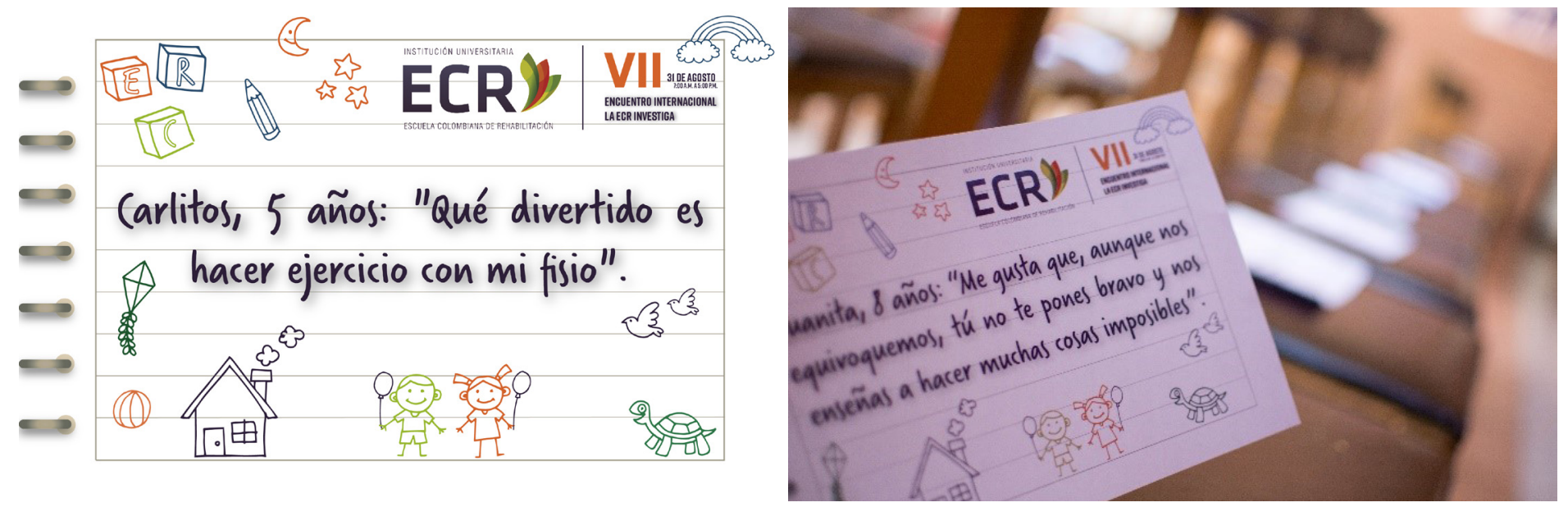

12. Docentes ECR. Línea de Investigación en Diversidad e Inclusión 


\section{Referencias}

Cortese, M., y Ferrari, M. (2003). Algunas reflexiones sobre el contrato fundacional y el mandato social de la escuela especial. En Educación Especial. pp. 16-33

Cosse, I., Llovet, V., Villalta, C., y Zapiola, M. (2011). Infancias: políticas y saberes en Argentina Brasil. Siglos XIX y XX.

Frigerio, G., Poggi, M y Tiramonti, G. (1994). Las instituciones Educativas. Cara y Ceca. Elementos para su comprensión. Buenos Aires: Troquel Educación - Serie Flacso 